\title{
G s. \\ Identification of Metabolic Reprogramming Related Gene Signature to Predict the Prognosis of Bladder Cancer Patients
}

\section{Tinghao Li}

The First Affiliated Hospital of Chongqing Medical University https://orcid.org/0000-0002-7980-6298 Hang Tong

The First Affiliated Hospital of Chongqing Medical University

Hubin Yin

The First Affiliated Hospital of Chongqing Medical University

\section{Honghao Cao}

Rongchang tranditional Chinese medicine hospital

\section{Junlong Zhu}

the first affliated hospital of Chongqing medical uiversity

\section{Zijia Qin}

The First Affiliated Hospital of Chongqing Medical University

\section{Siwen Yin}

The First Affiliated Hospital of Chongqing Medical University

\section{Weiyang He ( $\nabla$ weiyang1262020@126.com )}

The First Affiliated Hospital of Chongqing Medical University https://orcid.org/0000-0002-7445-8781

\section{Research article}

Keywords: Metabolic reprogramming, Prognosis, Bladder cancer, TCGA, GEO

Posted Date: December 23rd, 2020

DOI: https://doi.org/10.21203/rs.3.rs-132465/v1

License: (c) (1) This work is licensed under a Creative Commons Attribution 4.0 International License. Read Full License 


\section{Abstract}

Background: Different kinds of metabolic reprogramming have been widely researched in multifarious cancer types and show up as a guaranteed prognostic predictor, while bladder cancer (BLCA) is most frequent urothelium carcinoma but with poor prognosis despite there are emerging treatments, for lack of reliable predicting biomarkers to early predict the prognosis and delayed treatment options for patients in the terminal stage. Our study aims to explore new prognostic factors related to metabolism in BLCA and make these genes up as novel risk stratification.

Methods: We selected a large number of samples downloaded from TCGA (The Cancer Genome Atlas) to find out the possible glycolysis-related genes that correlated with differentiation from cancer sample to normal tissue, aimed to find out a more credible model. To make our signature more believable, we chose the clinical features information from GEO (Gene Expression Omnibus) database as external validation cohort.

Results: Finally, we established a three glycolysis-related gene signature based on the expression of AK3, GALK1 and NUP205 to make a prediction on the prognosis of BLCA patients, which were also validated by external cohort and whole mixed cohort. As a result, we built a three glycolysis-related gene signature and found its prognosis value is more valuable in high malignancy patients, which may help physicians to make a more aggressive choice.

\section{Introduction}

Bladder cancer (BLCA) causes the $7 \%$ morbidity of all cancers and $3 \%$ mortality of all cancer death $s^{[1]}$. Patients with BLCA they are divided into NMIBC (non-muscle invasive bladder cancer) and MIBC (muscle invasive bladder cancer) according to pathological grade. For BLCA especially MIBC is featured by high stake of invasion, metastasis and recurrence, the prognosis is still not optimistic though there are constantly emerging diverse treatment methods or establishing molecular pathology types ${ }^{[2,3]}$. The reason might be attributed to the heterogeneity of genomic, transcriptional and cellular of cancer cells, which contribute to the numerous and unpredictable outcomes of BLCA patients ${ }^{[4]}$. Therefore, it is crucial to develop an effective prediction model with the use of prognostic biomarkers to distinguish those patients with high risk of BLCA, particularly patients in terminal stages or named high malignancy, and make a preliminary prognosis prediction so that physicians could take more aggressive treatment measures earlier.

Malignant tumors are not only genetic diseases but also energy metabolic diseases. With the malignant behaviors of tumors particularly solid tumors like bladder cancer, they typically consume more nutrients than their bioenergy needs and divert metabolites to pathways that support biosynthetic platforms or alter metabolic patterns to adjust the tumor microenvironment which often consist of hostile factors like starvation, inflammation, hypoxia and so on, that is also called metabolic reprogramming ${ }^{[5-7]}$. There are three kinds of main nutrient substances named glucose, fatty acid and amino acid, and each of their 
metabolic reprogramming have been found to be closely associated with the malignant progression and is related to poor prognosis in BLCA and many malignant tumors. Nath et al. ${ }^{[8]}$ reported that fatty acid metabolic reprogramming via the alteration of related genes expression included CAV1, CD36, MLXIPL, CPT1C, CYP2E1 and so on could have an effect on the metastatic progression and associate with poor prognosis of human cancers. Lee et al ${ }^{[9]}$ also found that adjusting lipid metabolism is essential for establishment of cisplatin resistance in BLCA. Amino acid also played a crucial role in cancer cells which supports growth and reproduction of cells, while several researches discovered that with the abnormal convert or overexpression of some enzymes that correlated with amino acid metabolism like LAT1, there turned out a bad prognosis and significant progression in BLCA patients ${ }^{[10-12]}$. Turning oxidative phosphorylation into glycolysis even with sufficient oxygen is also known as Wurburg effect, which has been considered to be associated with malignant progression in most malignancies ${ }^{[13]}$. The correlation between aerobic glycolysis and malignant progression of bladder cancer and poor prognosis has been reported in many literatures, and glycolysis related genes could play as a prognostic role have also been reported in many articles. Tian et al. ${ }^{[14]}$ established a three-gene glycolytic signature, including MET, B3GNT3, and SPAG4, for risk stratification and prognosis prediction in Pancreatic ductal adenocarcinoma, Liu et al. ${ }^{[15]}$ also created nine glycolysis-related genes involving B3GALT6, PAM, LCT, GMPPB, GLCE, DCN, CAPN5, GYS2 and FBP2 to play the role of prognosis-related genes in endometrial cancer. Our group members had published before that they had successfully built a four glycolysis related genes signature as a prognostic model based on 19 pairs bladder cancer samples and their adjacent normal samples of BLCA patients ${ }^{[16]}$.

Now we are trying to find a more representative prediction model in a larger scale cohort which may correlated with the metabolic reprogramming based on the BLCA dataset from The Cancer Genome Atlas (TCGA), and we further divided different clinical characteristic BLCA patients into matched subgroups to make our prediction model in different clinical characteristics of the population more accurate. Finally we successfully established a three glycolysis-related gene signature that could forecast and assess the prognosis of BLCA patients, which was validated in the Gene Expression Omnibus (GEO) dataset and mixed whole cohort.

\section{Materials And Methods}

\section{Data collection}

The mRNA expression profiles and clinical information were downloaded from TCGA data portal as the training cohort. In the aggregate 349 samples consist of 331 bladder cancer and 18 healthy samples were achieved for the further study. GEO dataset of 165 BLCA patients was used as an external validation cohort, which was downloaded from GEO database ${ }^{[17]}$. The details of included cohorts' clinical characteristics are listed on Table 1. 
Table 1

Characteristics of The Cancer Genome Atlas and Gene

Expression Omnibus Databases in This Study

\begin{tabular}{|c|c|c|c|}
\hline Covariates & Category & TCGA dataset & GEO dataset \\
\hline All & / & 331 & 165 \\
\hline \multicolumn{4}{|l|}{ Gender } \\
\hline & Female & 85 & 30 \\
\hline & Male & 246 & 135 \\
\hline \multirow[t]{2}{*}{ Age (year) } & $\leq 65$ & 130 & 74 \\
\hline & $>65$ & 201 & 91 \\
\hline \multicolumn{4}{|l|}{ T stage } \\
\hline & $\mathrm{T}_{0-2}$ & 100 & 135 \\
\hline & $\mathrm{T}_{3-4}$ & 205 & 30 \\
\hline \multicolumn{4}{|l|}{$\mathrm{N}$ stage } \\
\hline & $\mathrm{N}_{0}$ & 191 & 149 \\
\hline & $\mathrm{N}_{1-3}$ & 104 & 16 \\
\hline \multicolumn{4}{|l|}{ M stage } \\
\hline & $\mathrm{M}_{0}$ & 157 & 158 \\
\hline & $M_{1}$ & 8 & 7 \\
\hline \multicolumn{4}{|l|}{ AJCC stage } \\
\hline & Stage1-2 & 108 & 129 \\
\hline & Stage3-4 & 222 & 36 \\
\hline \multicolumn{4}{|c|}{ Survival status } \\
\hline & Alive & 228 & 68 \\
\hline & Deceased & 102 & 97 \\
\hline
\end{tabular}

\section{Gene set enrichment analysis (GSEA)}

Analysis were presented with the use of GSEA software 3.0 to identify the hallmark gene sets of different metabolic ways provided by Molecular Signatures Database (MSigDB), to predict biological processes 
between 331 bladder cancer and 18 healthy samples from TCGA cohort. Normalized enrichment score $(\mathrm{NES})>1.6$, nominal $p$-value $<0.05$ and false discovery rate $(F D R) q$-value $<0.05$ were set as the cutoff and were chosen for core genes.

\section{Establishment of metabolism-related gene signature as prognostic model}

Then we used univariate Cox regression analysis to screen out prognosis-associated genes that related to metabolism. Hazard ratio (HR) $<1$ represents well overall survival (OS) outcomes, and HR $>1$ represents poor OS outcomes. We chose genes with $\mathrm{P}<0.05$ as prognosis related metabolic genes and utilized for the multivariate Cox regression analysis. We used the multivariate Cox regression analysis finally created a three glycolysis-related gene signature, the risk score formula according to follows: Risk score $=\sum_{i=1}^{\mathrm{n}}$ $\beta 1$ *expression $\left(G_{i}\right)^{[18]}$. In this formula, " $n$ " means the number of prognostic genes, "expression $\left(G_{i}\right)$ " means the expression of gene $i$, and " $\beta i$ " means the regression coefficient of the corresponding gene obtained by the multivariate Cox regression model. BLCA patients were split into high risk or low risk subgroups by the median risk score.

\section{Validation of the efficacy of the prognostic three glycolysis- related gene signature}

The prognostic three glycolysis-related gene signature was brought in the external validation cohort and the whole cohort next. Patients in the validation cohort and whole cohort were divided into high or low risk groups depending on the median risk score from the training cohort. Kaplan-Meier curve analysis, 3and 5-years time-dependent ROC analysis, and risk scores of each patient distribution in different subgroups of clinical characteristics were then performed.

\section{Protein-protein interaction (PPI) network construction}

We used a website named GeneMANIA (http://www.genemania.org/) to forecast the interactions and functions of genes, and build the network and single out hub genes in the regulatory network including physical interaction, co-expression and so on.

\section{Estimation of clinical independence and construction of the nomogram}

Then we eliminated BLCA patients with lacking exactly clinicopathological information like TNM stage or AJCC (American Joint Committee on Cancer) stage in training cohort. We utilized the "rms" R package (version 3.5.1) to integrate clinical features with the risk scores of our three glycolysis-related gene signature, to construct a nomogram for further clinical prediction. Then we built the calibration plots and time-dependent receiver operating characteristic (ROC) curves to evaluate the performance of our nomogram. 


\section{Results}

\section{Identification metabolism-related genes using GSEA}

In the aggregate 409 samples including 391 BLCA and 18 healthy samples with their clinical features information were achieved from the TCGA as training cohort and 165 BLCA samples achieved from the GEO as external validation cohort (Table 1). We integrated mRNA expression profiles and clinical information, and the limma algorithm was used to identify the mRNAs that were differentially expressed in the BLCA samples relative to healthy samples in training cohort, there were 132 up-regulated and 33 down-regulated genes with respect to healthy samples were obtained $(p<0.05)$. GSEA was used to explore whether there were significant genomic differences between BLCA and normal tissues in different kinds of metabolic phenotypes. Finally we found that these genes are significantly enriched in amino acid (ACID_AMINO_ACID_LIGASE_ACTIVITY), fatty acid (GO_FATTY_ACID_BETA_OXIDATION) and glycolysis metabolism. Glycolysis was the most obvious pathway in two gene sets (HALLMARK_GLYCOLYSIS and REACTOME_GLYCOLYSIS)(Fig. 1). Then we analyzed different expressed mRNAs and found in total 151 participating genes, including 109 genes in HALLMARK_GLYCOLYSIS gene sets (NES $=1.71$, nominal $\mathrm{P}<$ 0.005 , FDR $<0.005$ ) and 42 in REACTOME_GLYCOLYSIS (NES $=1.62$, nominal $P<0.05$, FDR $<0.05$ ) respectively.

\section{Construction of glycolysisrelated genes and BLCA prognosis models}

Univariate Cox regression analysis were used to investigate the relationship between Glycolysis and the prognosis of BLCA patients as preliminary sizing, of which four genes with the cut off $P<0.05$ were primary selected. Then used multivariate Cox regression analysis to examine the relevance between the expression of four genes and the survival of patients. Of which we built the prognostic model constructed with a protective role (Arginine kinase $3, A K 3$ ) with $\mathrm{HR}<1$ with lower expression and a risk role (Galactokinase 1, GALK1 and nucleoporins 205, NUP205) with HR > 1 with higher expression (Fig. 2a), and the risk score formula came out as the followed: $-0.33227^{*} \mathrm{AK} 3+0.462604^{*}$ GALK1 $+0.539478^{*}$ NUP205.

BLCA patients were ranked by their risk scores and split into a high risk group $(n=165)$ and a low risk group ( $n=166)$ using the median risk score of the series (Fig. 2b). As shown in the Fig. 2c, a higher death rate was notable for high risk group than for those with low risk scores. In the model, low risk group had significantly longer overall survival time than high risk group rendered by a Kaplan-Meier survival curve and a log-rank test (Fig. 2d). Then we used our prognosis model with the ROC curve analysis (Fig. 2e), containing the 3, 5-year survival of the proportions under the receiver operating characteristic curve (AUC) which were 0.651 and 0.709 respectively, to verify the sensitivity and specificity in predicting survival for BLCA patients. We used both univariate and multivariate Cox regression analyses to assess whether our prognosis model was an independent predictor of BLCA. The results revealed a moderate and 
independent prognostic power for risk scores (Fig. 2f,g), which further validated the dependability of our previous prognostic model.

\section{Hierarchical analysis between the clinical characteristics and the three glycolysis-related gene signature}

The results of univariate and multivariate Cox regression analyses turned out obviously differences between the high and low risk groups associated with risk scores, age, clinicopathology and cancer stage according to the AJCC (Fig. 2f,g). On the grounds of our univariate Cox regression analyses results, we used stratification analysis to explored the relationship between the overall survival and different clinical features, and found that age, tumor status including $T$ stage, $N$ stage and $M$ stage and stage according to our three glycolysis-related gene signature were significantly related to the survival, which were rendered by a Kaplan-Meier survival curve and a log-rank test (Fig. 3a-e). Next we classified the patients into different subgroups with median risk core by our signature according to age ( $\leq 65$ to $>65$ ), T stage (T0-2 to T3-4), N stage (N0 to N1-3), M stage (M0 to M1-3) and AJCC stage (stage I-II to stage III-IV). As shown in the Fig. 3f-j and Sfig. 1a-e (Additional files), Kaplan-Meier curves showed that in the training cohort patients with high-risk scores brought out poorer prognosis in $\mathrm{T}_{3-4}$ subgroup, N0 subgroup, M0 subgroup, higher stage and elder $($ age $>65)$ patients $(P<0.05)$ relative to the other group, which suggested that our three glycolysis-related gene signature may have a better prognostic value in higher malignancy BLCA patients.

\section{Validation of the three glycolysis-related gene signature for survival prediction}

Next we verified our prognosis model in training cohort and whole cohort to further confirm its reliability. We analyzed expression levels of three glycolysis-related genes in both the validation cohort and whole cohort, respectively, GALK1 and NUP205 were expressed higher in the high risk group while AK3 was expressed lower (Fig. 4a,b). To validate the survival prediction of the three glycolysis-related gene signature, risk scores were calculated for each patient in both the validation cohort and whole cohort. As the risk score formula mentioned before, BLCA patients were ranked and split into a high risk group or a low risk group using the median risk score from the training cohort as the cutoff point (Fig. 4c,d). As expected, a higher death rate was noted for BLCA patients with high risk scores than for those with low risk scores in each cohort (Fig. 4e,f). In accord with our training cohort results, low risk patients revealed longer overall survival time than high risk patients in both validation cohort and whole cohort (Fig. 4g,h) as $p<0.001$ in each cohort, ROC curve analysis also showed independent prognostic power of our model (Fig. 4i,j). Then we further analyzed the association between our three glycolysis-related gene signature and the clinicopathological features of BLCA in the validation cohort. In accordance with our prediction before, results indicated that our model is notably related to older, higher clinicopathological stage and AJCC stage, suggesting the signature may play a crucial role in BLCA malignant progression (Fig. 4k-o). Then we analyzed the association between various clinical features and survival in our validation group. Kaplan-Meier curves also showed that in the validation cohort patients with progression 
clinicopathological features brought out poorer prognosis in $T_{3-4}$ subgroup, $N_{1-3}$ subgroup, $M_{1-3}$ subgroup, higher stage and elder (age $>65)$ patients $(P<0.05)($ Sfig. $f-j)$, which also confirmed that our model possess a more accurate prognostic value for higher malignancy BLCA patients.

\section{Genetic information of the three glycolysisrelated genes and validation of the expression levels}

The frequently altered, or said mutated, sites of the three glycolysis-related genes and its frequency of mutation were analyzed by cBioPortal software (Fig. 5a,b). The network including AK3, GALK1, NUP205 and their most interacted neighbor genes were constructed by GeneMANIA (Fig. 5c). As shown in the picture, there were 23 genes including three target genes were automatic generated by GeneMANIA, consisted of physical interaction, co-expression and so on. To eliminate the inner relationship of the three hub-genes, we analyzed the correlation of the three genes and found that the absolute values of the correlation coefficient among them were all less than 0.3 , indicated that there were no correlation among these three genes (Fig. 5d).

After that we also investigated the expressions of these three genes in normal and BLCA tissues. As illustrated in Fig. 5e, there were significant different expression levels between normal and cancer tissues. Immunohistochemistry $(\mathrm{IHC})$ staining images were downloaded from the Human Protein Atlas database (https://www.proteinatlas.org/) to verify the expression. Images showed that the expression of AK3 in urothelial normal tissue was stronger than that in urothelial carcinoma tissue, while the intensity of GALK1 performs on the contrary (Fig. $5 f, g$ ), which also confirmed that in our signature based on three glycolysis-related genes there were both risk factors and protective markers to make a more accuracy prognostic model. But there were no data about IHC staining images of NUP205, which remained a defectiveness to be further investigated.

\section{Nomogram establishment and validation}

Then we built a nomogram to evaluate the possibility of the 3- and 5-year OS and establish a clinically method to predict the survival probability of BLCA patients based on the training cohort. Seven independent predictors including age, grade, AJCC stage, T-stage, N-stage, M-stage and risk score were incorporated to build the nomogram (Fig. 6a). The calibration plots indicated satisfying coherence between the nomogram predictions and actual observations (Fig. 6b, c). ROC curve analysis also showed the AUC of the nomogram at 3 - and 5-year were 0.707 and 0.76 (Fig. $6 \mathrm{~d}$ ).

\section{Discussion}

As one kind of solid tumors, bladder cancer has come to be a common malignant tumor that threatens human lives. With the hostile environment where solid tumor cells living in like starvation, hypoxia and so on, tumor cells may exchange its patterns of metabolism to adjust the microenvironment which is also called metabolic reprogramming ${ }^{[19,20]}$. It has widely believed that metabolic reprogramming is one of the hallmarks of cancer cells ${ }^{[19,20]}$, while there were so many findings aimed to seek out how could it 
happens and effect on the tumor malignent behaviors ${ }^{[22,23]}$, our group tried to draw our attention on the prognostic role of metabolic reprogramming. As the report our group members had published before, they had successfully built a four glycolysis related gene signature as a prognostic model based on 19 pairs bladder cancer samples and their adjacent normal samples of BLCA patients ${ }^{[24]}$. Now we are trying to find out a more representative prediction model from a larger cohort and we successfully made it from a training cohort with 349 samples including BLCA samples and normal tissues downloaded from TCGA data base and 165 BLCA samples from GEO data base. After integrated the transcriptional expression of training cohort and enriched via GSEA in different metabolic gene sets, we finally found that glycolysis is most significantly correlated with the prognosis of BLCA. Glycolysis is a common metabolic pathway in cells and its reasonably to associat with the development of $\mathrm{BLCA}^{[25]}$. In our study, we performed a bioinformatic analysis to identify metabolic related genes involved in BLCA based on the different expression genes between BLCA samples and normal tissues, and there were 132 up-regulated and 33 down-regulated genes obtained. With the use of multivariate Cox regression analyses and univariate Cox regression analyses we finally developed a three glycolysis-related gene signature (AK3, GALK1 and NUP205) for prognosis prediction of BLCA. In an attempt to further validate its prognostic value in GEO data and whole cohort, we also got the consistent results, which imply that this three-gene signature could act as a prognostic indicator in BLCA.

We found these three genes are connected with tumors or even BLCA through reviewed the existing literature. Among our signature, AK3 is located on chromosome 9 and mainly functions in the mitochondrial matrix, has been demonstrated as tumor suppressor function in many other solid tumors except BLCA ${ }^{[26]}$. Melle et al. ${ }^{[27]}$ found out that AK3 could play as a tumor suppression role in hepatocellular carcinoma and its low expression was associated with tumor development and progression. Although there were little research about AK3 on BLCA patients' prognosis, Chang. et al. ${ }^{[28]}$ had reported that in urothelial cells AK3 could help to enhance the sensitivity of bladder cancer cells to cisplatin, while down-regulation of AK3 by cigarette smoke condensate would contribute to establishment of drug resistance. And the suppress role of AK3 in BLCA has also been validated in our study, but it still needs further exploration on how it works and what facilitate the down-regulation happens. GALK1 is associated with metabolism of galactose absorption from digested foodstuffs, as well as that produced endogenously. Tang et al. ${ }^{[29]}$ discovered the role of GALK1 in hepatocellular carcinoma, reported that inhibition of GALK1 via siRNA could suppress the growth of cancer cells, and they also found out the possibility of the interaction between galactose metabolic and PI3K/AKT pathways. However, there was no report about the function of GALK1 in BLCA, it remains enormous potential to find it's role as an oncogene. NUP205 is a widely researched oncogene that could conduce to modulate the timing of mitotic onset ${ }^{[30]}$. Xiong et al. ${ }^{[30]}$ reported that NUP205 is associated with clinical outcomes of hepatocellular carcinoma patients via the crosstalk between hsa_circ_0088364 and hsa_circ_0090049. Our group members had also published its prediction role in BLCA patients as one of oncogenes in their signature before $^{[24]}$, while it showed up again in our model to make us more firmly believe that it has the potential to play as a predictor of prognosis for BLCA patients, with malignancy subgroups in our research. 
Glycolysis-related prognostic predict models counting on core glycolysis-related genes with different clinical characteristics have been indicated to serve as reliable prognostic indicators in various solid tumors $^{[14-16]}$. We selected a lager number of samples to find out the possible glycolysis-related genes that correlated with differentiation from cancer sample to normal tissue, aimed to find out a more credible model. To make our signature more believable, we chose the clinical features information from GEO data base as external validation cohort.

In the present study, we also found that our model were more valuable in people with worse clinical characteristics like older or higher malignancy, which were also confirmed by our validation group. Our three glycolysis-related gene signature could accurately categorize BLCA patients into high or low risk subgroups which represented patients' outcomes. Our study also built a comprehensive nomogram that incorporated our model with different clinical features including age, gender, TNM stage and AJCC stage to effectively predict the survival of BLCA patients. Our prognostic scoring system could provide a higher accuracy method to help physicians to make a more individualized estimation of survival or more aggressive treatment options to patients especially those in late stage.

\section{Conclusion}

In summary, our study find out a three glycolysis-related gene signature to make a prediction on the prognosis of BLCA patients based on the expression of AK3, GALK1 and NUP205, which were also validated by external cohort from GEO data base and whole cohort. Our findings would contribute to revealing novel potential molecular therapeutic targets while there were just a few studies about these three genes and its effect on the bladder cancer which may need to be further researched. Also, independent cohort studies and experimental studies may need further performed to validate the robustness and practicability of our model in BLCA prognosis prediction.

\section{Abbreviations}

BLCA: bladder cancer; TCGA: The cancer genome atlas; GSEA: Gene set enrichment analysis; GEO: Gene expression omnibus; NMIBC: non-muscle invasive bladder cancer; MIBC :muscle invasive bladder cancer; MSigDB: Molecular Signatures Database; NES: Normalized enrichment score; FDR: False discovery rate; HR: Hazard ratio; OS: overall survival; PPI: Protein-protein interaction; AJCC: American Joint Committee on Cancer; ROC: Receiver operating characteristic; AUC: operating characteristic curve; IHC: Immunohistochemistry; AK3: Arginine kinase 3; GALK1: galactokinase 1; NUP205: nucleoporins 205.

\section{Declarations}

\section{Ethical Approval and Consent to Participate}

This article meets the conformances to requirements of TCGA and GEO websites of publishment, and does not contain any studies with human participants or animals performed by any of the authors. 


\section{Consent for Publication}

Not applicable.

\section{Competing interests}

There are no financial disclosures or competing interests among authors.

\section{AUTHORS' CONTRIBUTIONS}

LTH conceived and designed the study. QZJ and YSW collected the data. CHH and ZJL interpreted and analyzed the data. $\mathrm{LTH}, \mathrm{TH}$, and YHB wrote the manuscript. QZJ revised the manuscript critically.

\section{ACKNOWLEDGMENTS}

This study was supported by funds from the National Natural Science Foundation of China (Grant N0.81874092)

\section{References}

1. KAMAT A M, HAHN N M, EFSTATHIOU J A, et al. Bladder cancer[J]. Lancet, 2016,388(10061): 27962810.

2. DALANGOOD S, ZHU Z, MA Z, et al. Identification of glycogene-type and validation of ST3GAL6 as a biomarker predicts clinical outcome and cancer cell invasion in urinary bladder cancer[J]. Theranostics, 2020,10(22): 10078-10091.

3. LIAN P, WANG Q, ZHAO Y, et al. An eight-long non-coding RNA signature as a candidate prognostic biomarker for bladder cancer[J]. Aging (Albany NY), 2019,11(17): 6930-6940.

4. MEEKS J J, AL-AHMADIE H, FALTAS B M, et al. Genomic heterogeneity in bladder cancer: challenges and possible solutions to improve outcomes[J]. Nat Rev Urol, 2020,17(5): 259-270.

5. LOZY F, KARANTZA V. Autophagy and cancer cell metabolism[J]. Semin Cell Dev Biol, 2012,23(4): 395-401.

6. DEBERARDINIS R J, LUM J J, HATZIVASSILIOU G, et al. The biology of cancer: metabolic reprogramming fuels cell growth and proliferation[J]. Cell Metab, 2008,7(1):11-20.

7. WU X, ZHOU Z, XU S, et al. Extracellular vesicle packaged LMP1-activated fibroblasts promote tumor progression via autophagy and stroma-tumor metabolism coupling[J]. Cancer Lett, 2020,478: 93106.

8. NATH A, CHAN C. Genetic alterations in fatty acid transport and metabolism genes are associated with metastatic progression and poor prognosis of human cancers[J]. Sci Rep, 2016,6: 18669.

9. LEE M Y, YEON A, SHAHID M, et al. Reprogrammed lipid metabolism in bladder cancer with cisplatin resistance[J]. Oncotarget, 2018,9(17): 13231-13243. 
10. KAIRA K, SUNOSE Y, ARAKAWA K, et al. Prognostic significance of L-type amino-acid transporter 1 expression in surgically resected pancreatic cancer[J]. Br J Cancer, 2012,107(4): 632-638.

11. MAIMAITI M, SAKAMOTO S, YAMADA Y, et al. Expression of L-type amino acid transporter 1 as a molecular target for prognostic and therapeutic indicators in bladder carcinoma[J]. Sci Rep, 2020,10(1): 1292.

12. BACCI M, LORITO N, IPPOLITO L, et al. Reprogramming of Amino Acid Transporters to Support Aspartate and Glutamate Dependency Sustains Endocrine Resistance in Breast Cancer[J]. Cell Rep, 2019,28(1): 104-118.

13. GATENBY R A, GILLIES R J. Why do cancers have high aerobic glycolysis?[J]. Nat Rev Cancer, 2004,4(11): 891-899.

14. TIAN G, LI G, LIU P, et al. Glycolysis-Based Genes Associated with the Clinical Outcome of Pancreatic Ductal Adenocarcinoma Identified by The Cancer Genome Atlas Data Analysis[J]. DNA Cell Biol, 2020,39(3): 417-427.

15. LIU J, LI S, FENG G, et al. Nine glycolysis-related gene signature predicting the survival of patients with endometrial adenocarcinoma[J]. Cancer Cell Int, 2020,20: 183.

16. JIANG P, SUN W, SHEN N, et al. Identification of a metabolism-related gene expression prognostic model in endometrial carcinoma patients[J]. BMC Cancer, 2020,20(1): 864.

17. RIESTER M, TAYLOR J M, FEIFER A, et al. Combination of a novel gene expression signature with a clinical nomogram improves the prediction of survival in high-risk bladder cancer[J]. Clin Cancer Res, 2012,18(5): 1323-1333.

18. CHEN K, HE Y, LIU Y, et al. Gene signature associated with neuro-endocrine activity predicting prognosis of pancreatic carcinoma[J]. Mol Genet Genomic Med, 2019,7(7): e729.

19. HUI L, CHEN Y. Tumor microenvironment: Sanctuary of the devil[J]. Cancer Lett, 2015,368(1): 7-13.

20. XUE M, CHEN W, XIANG A, et al. Hypoxic exosomes facilitate bladder tumor growth and development through transferring long non-coding RNA-UCA1[J]. Mol Cancer, 2017,16(1): 143.

21. HANAHAN D, WEINBERG R A. The hallmarks of cancer[J]. Cell, 2000,100(1): 57-70.

22. LOGOTHETI S, MARQUARDT S, GUPTA S K, et al. LncRNA-SLC16A1-AS1 induces metabolic reprogramming during Bladder Cancer progression as target and co-activator of E2F1[J]. Theranostics, 2020,10(21): 9620-9643.

23. GIACOMINI I, RAGAZZI E, PASUT G, et al. The Pentose Phosphate Pathway and Its Involvement in Cisplatin Resistance[J]. Int J Mol Sci, 2020,21(3).

24. ZHANG C, GOU X, HE W, et al. A glycolysis-based 4-mRNA signature correlates with the prognosis and cell cycle process in patients with bladder cancer[J]. Cancer Cell Int, 2020,20: 177.

25. TAO T, SU Q, XU S, et al. Down-regulation of PKM2 decreases FASN expression in bladder cancer cells through AKT/mTOR/SREBP-1c axis[J]. J Cell Physiol, 2019,234(3): 3088-3104.

26. QIN W Y, FENG S C, SUN Y Q, et al. MiR-96-5p promotes breast cancer migration by activating MEK/ERK signaling[J]. J Gene Med, 2020,22(8): e3188. 
27. MELLE C, ERNST G, SCHEIBNER O, et al. Identification of specific protein markers in microdissected hepatocellular carcinoma[J]. J Proteome Res, 2007,6(1): 306-315.

28. CHANG X, RAVI R, PHAM V, et al. Adenylate kinase 3 sensitizes cells to cigarette smoke condensate vapor induced cisplatin resistance[J]. PLoS One, 2011,6(6): e20806.

29. TANG M, ETOKIDEM E, LAI K. The Leloir Pathway of Galactose Metabolism - A Novel Therapeutic Target for Hepatocellular Carcinoma[J]. Anticancer Res, 2016,36(12): 6265-6271.

30. HACHET V, BUSSO C, TOYA M, et al. The nucleoporin Nup205/NPP-3 is lost near centrosomes at mitotic onset and can modulate the timing of this process in Caenorhabditis elegans embryos[J]. Mol Biol Cell, 2012,23(16): 3111-3121.

31. XIONG D D, FENG Z B, LAI Z F, et al. High throughput circRNA sequencing analysis reveals novel insights into the mechanism of nitidine chloride against hepatocellular carcinoma[J]. Cell Death Dis, 2019,10(9): 658.

\section{Figures}


Figure 1 :

A Enrichment plot: GO_FATTY_ACID_BETA_OXIDATION

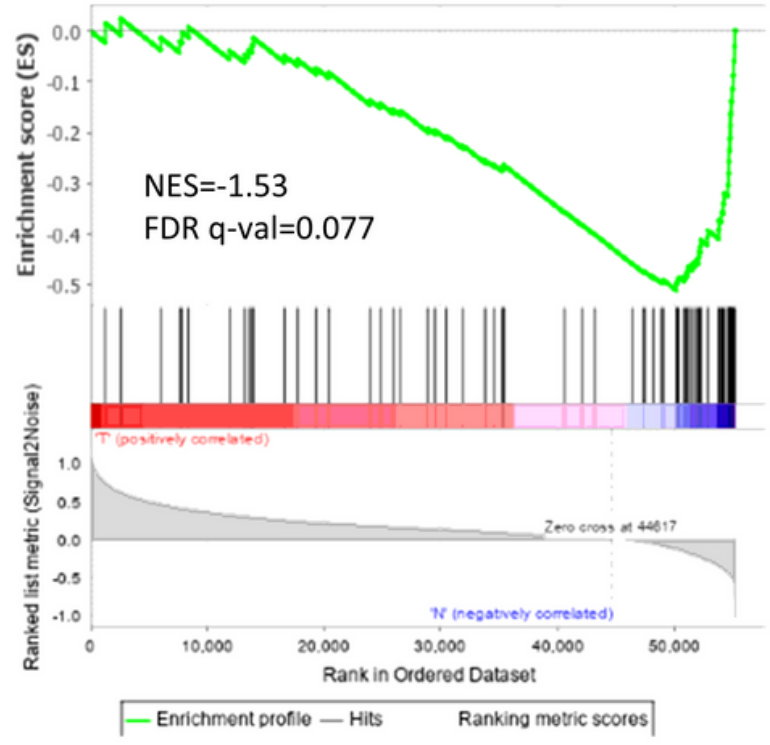

C

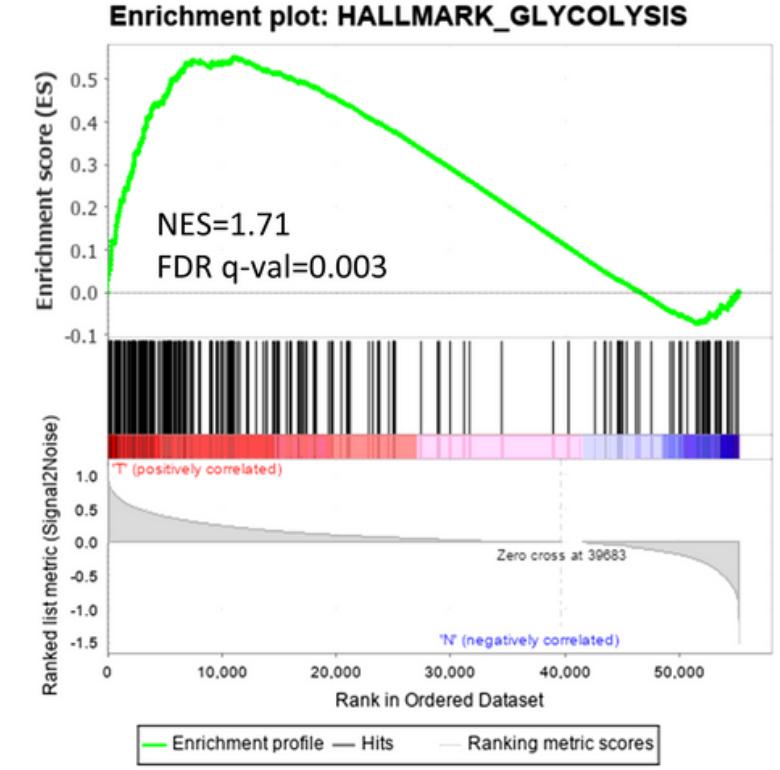

B Enrichment plot:ACID_AMINO_ACID_LIGASE_ACTIVITY

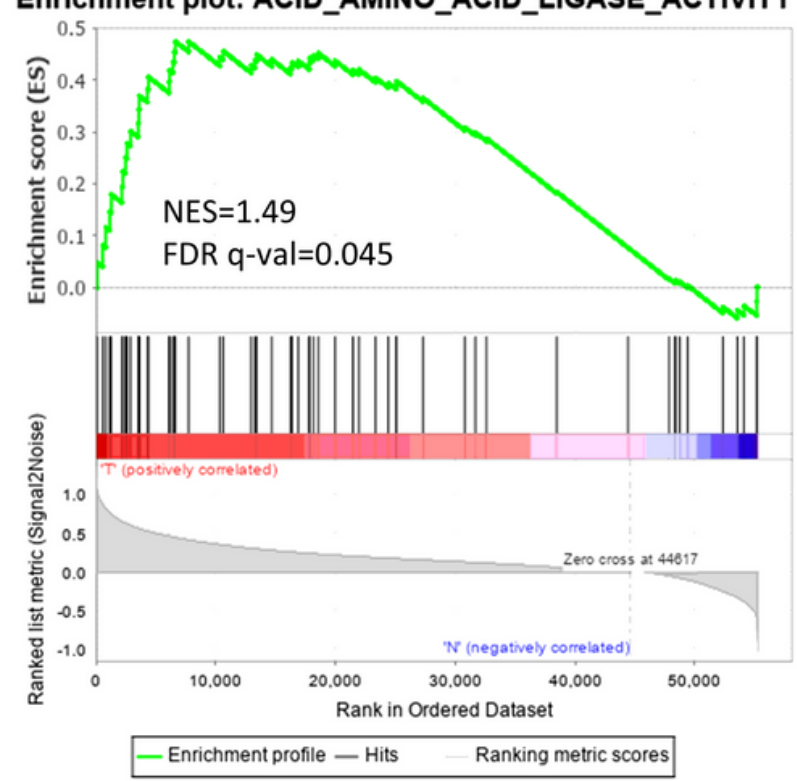

D

Enrichment plot: REACTOME_GLYCOLYSIS

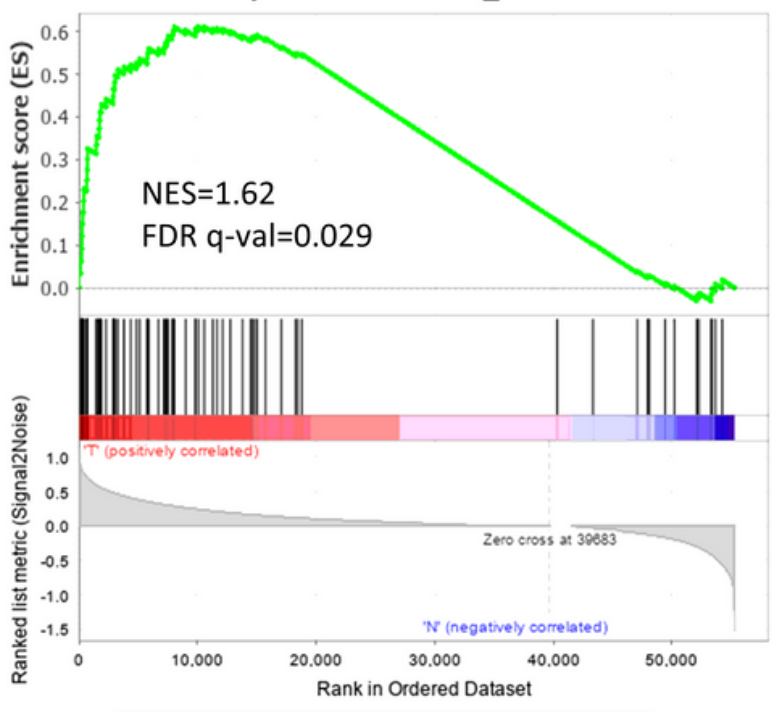

- Enrichment profile — Hits — Ranking metric scores

\section{Figure 1}

Gene set enrichment analysis (GSEA) of four metabolism-related gene sets (HALLMARK_GLYCOLYSIS,REACTOME_GLYCOLYSIS,GO_FATTY_ACID_BETA_OXIDATION, ACID_AMINO_ACID_LIGASE_ACTIVITY) that differentiated in bladder cancer (BLCA) and normal tissues based on The Cancer Genome Atlas (TCGA). 
Figure 2 :
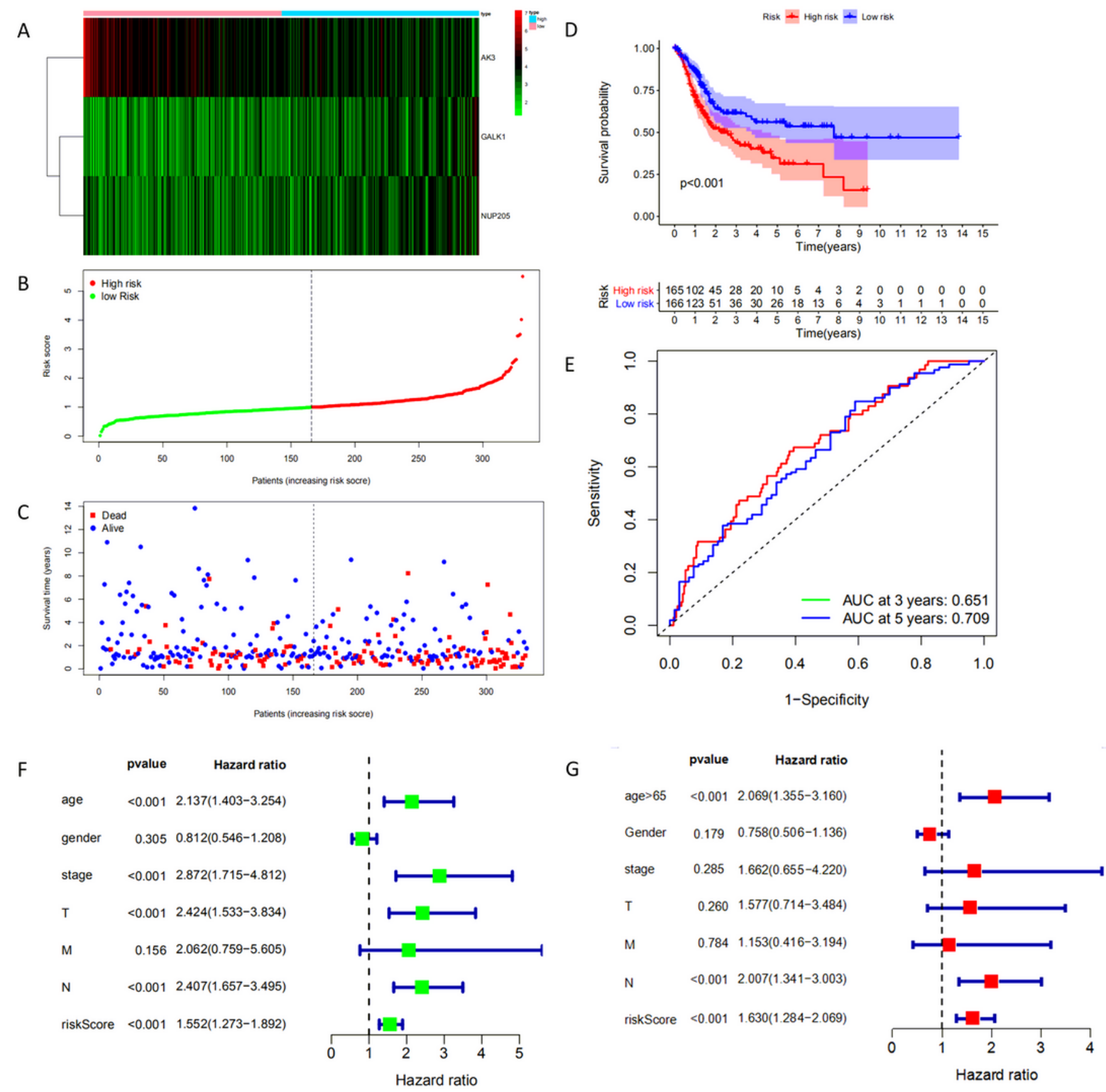

\section{Figure 2}

Risk score and prognostic analysis of the three glycolysis related genes model in the TCGA training cohort. a) Expression heatmap of the 3-mRNA signature in each patient. b) The risk score, c) survival status of the prognostic model for the training cohort. d) Kaplan-Meier curves of OS and e) timedependent ROC curves for prediction of 3- and 5-year OS. f, g) Univariate and multivariate Cox regression analyses for the risk score and each clinical characteristics. 
Figure 3 :
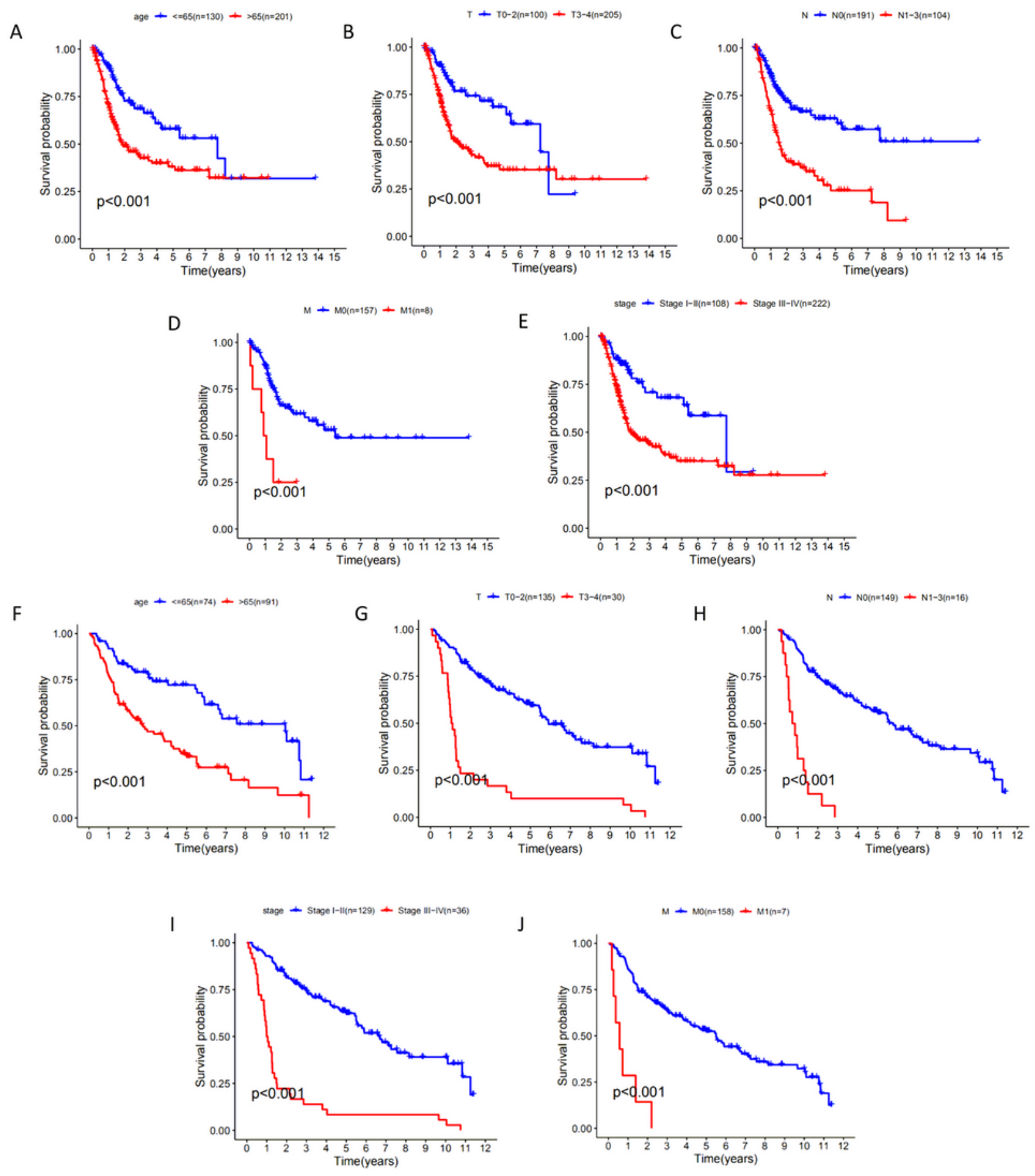

\section{Figure 3}

Hierarchical survival analysis with clinical characteristics based on Kaplan-Meier about a) age, b-d) TNM stage and e) the American Joint Committee on Cancer (AJCC) stage. Survival time of patients in high risk and low risk group indifferent subgroups like f) age, g) T3-4 stage, h) N0 stage, i) M0 stage and AJCC stage. 
Figure 4 :

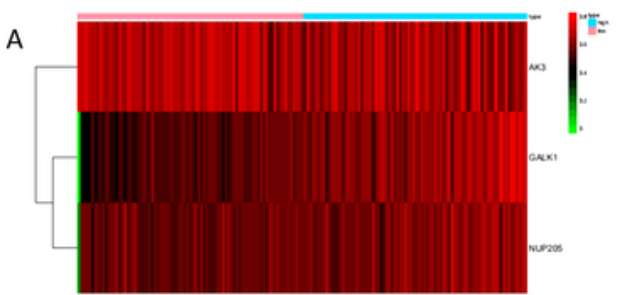

B
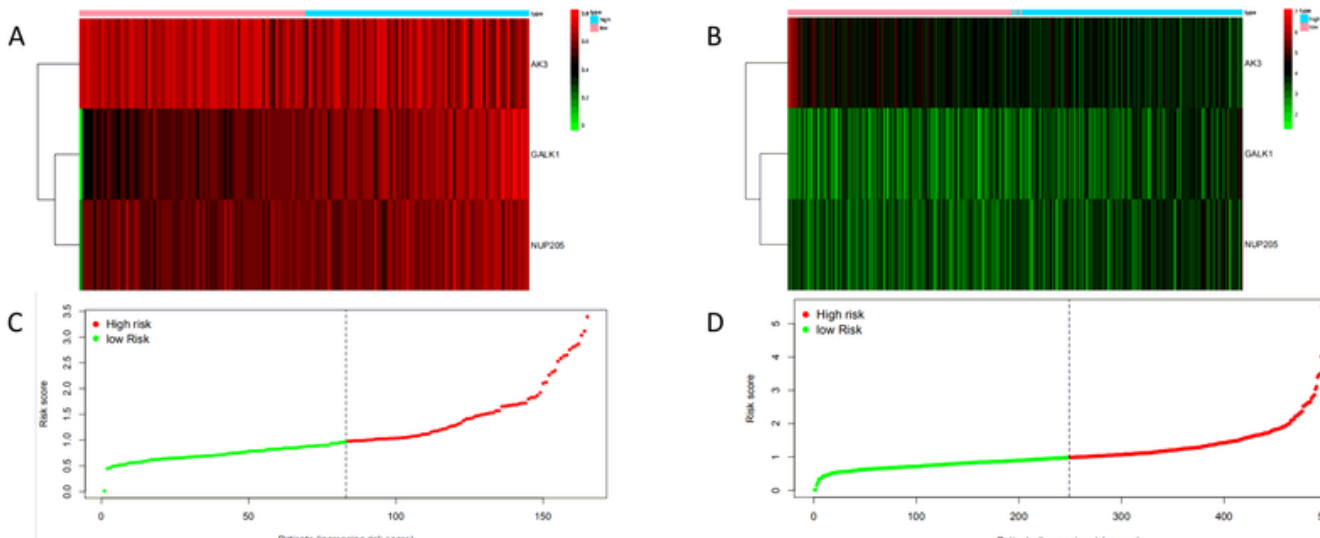

D
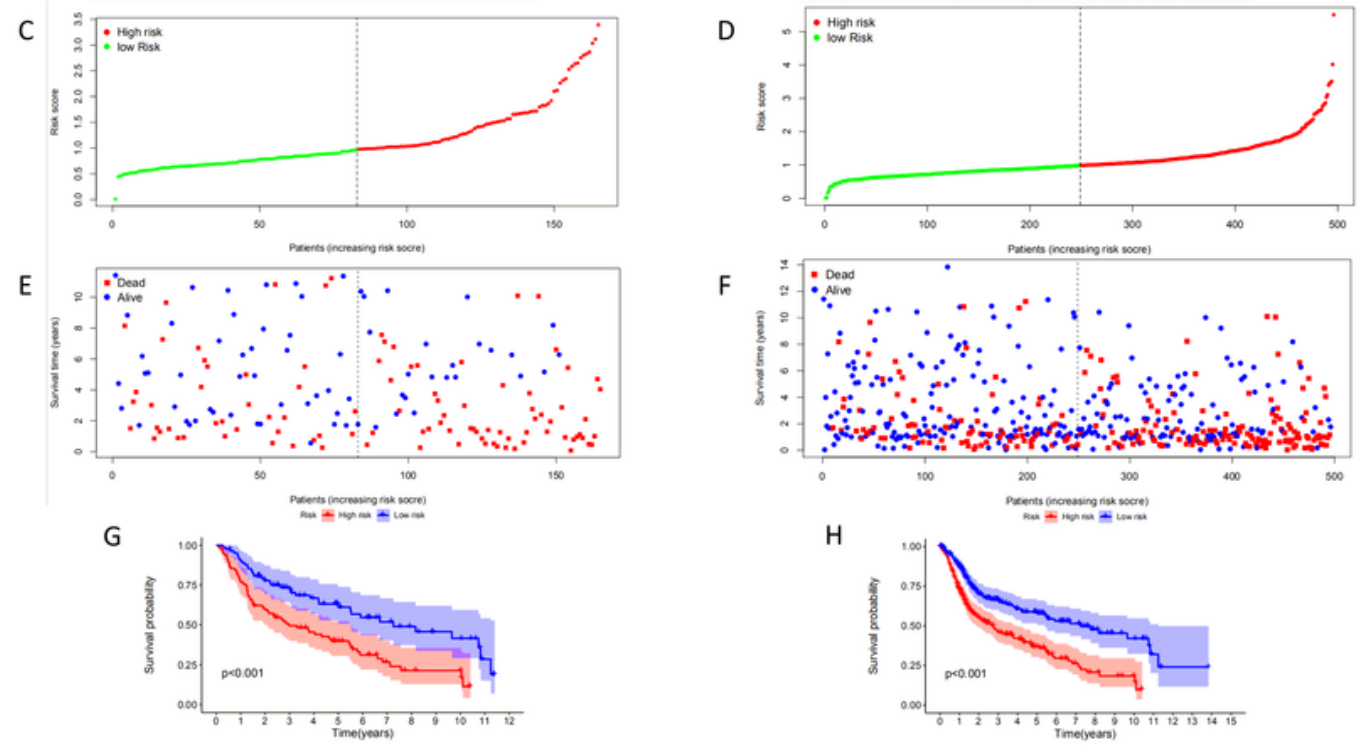

$\mathrm{H}$

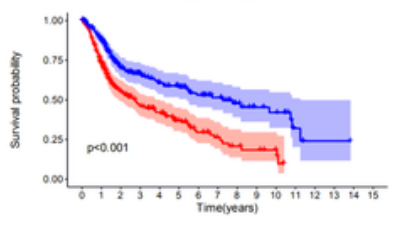

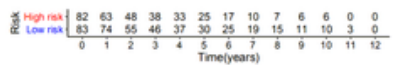

I

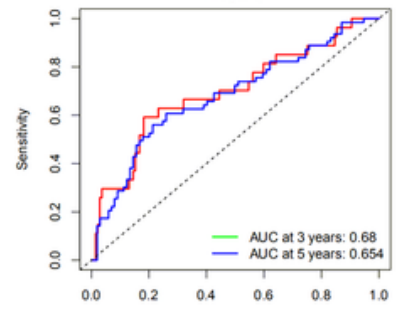

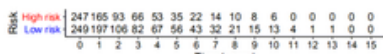
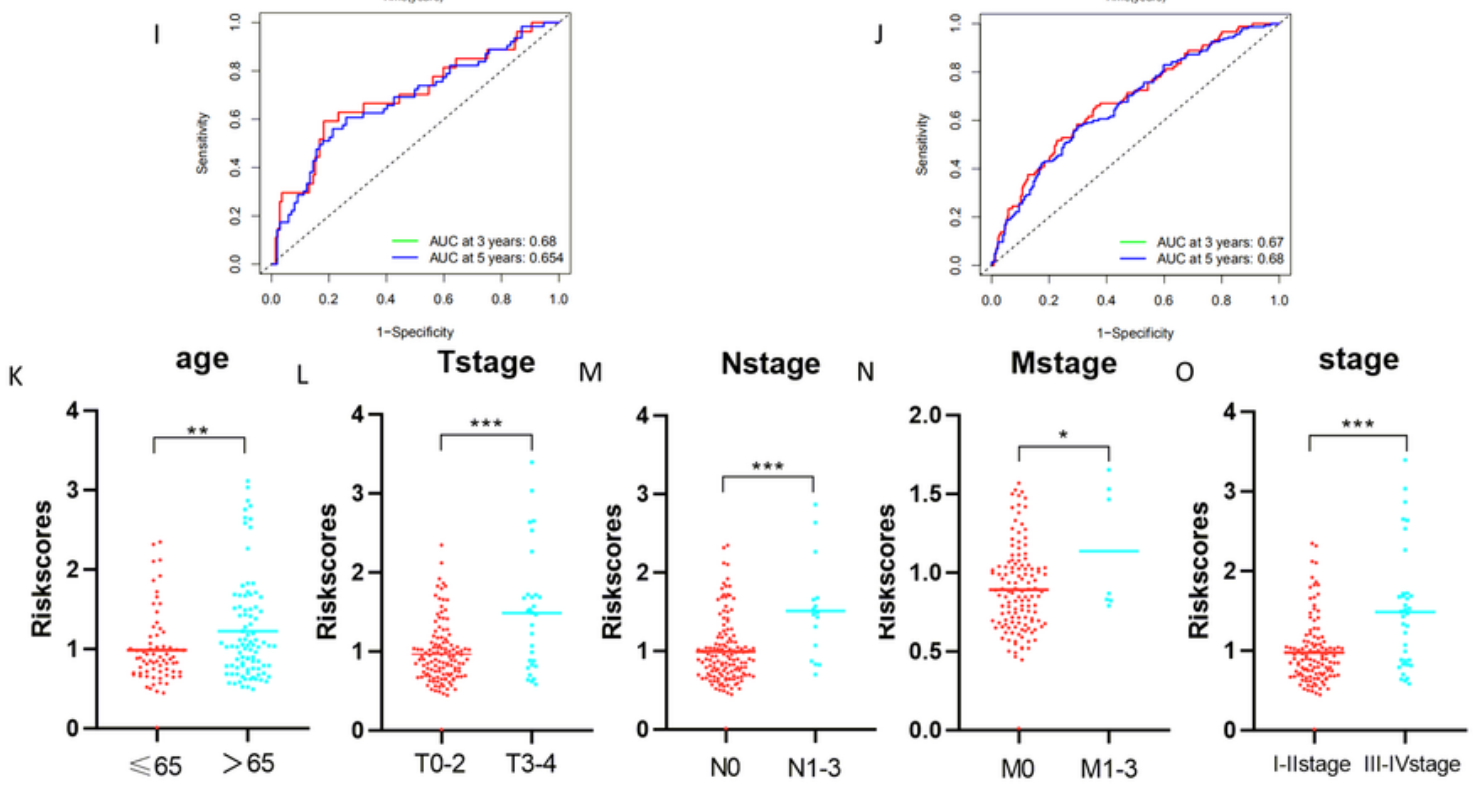

\section{Figure 4}

Validation of the efficacy of the risk model in validation cohort and entire cohort. a,b) The expression heatmap, c,d) survival status, e,f) risk score, g,h Kaplan-Meier survival, and i,j) time-dependent ROC curves of the prognostic model. Stratified analysis of different risk scores with clinical characteristics based on k) age, I-n) TNM stage and o) the AJCC stage. 
Figure 5 :

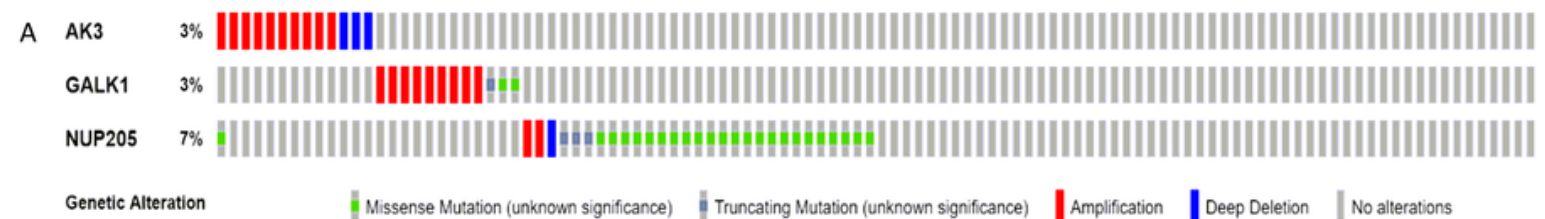

Genetic Alteration || Missense Mutation (unknown significance) || Truncating Mutation (unknown significance) |Amplification | Deep Deletion || No alterations

B AK3

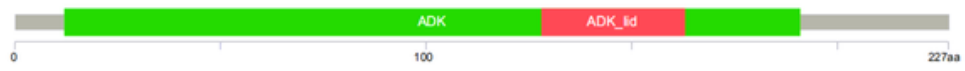

sen:
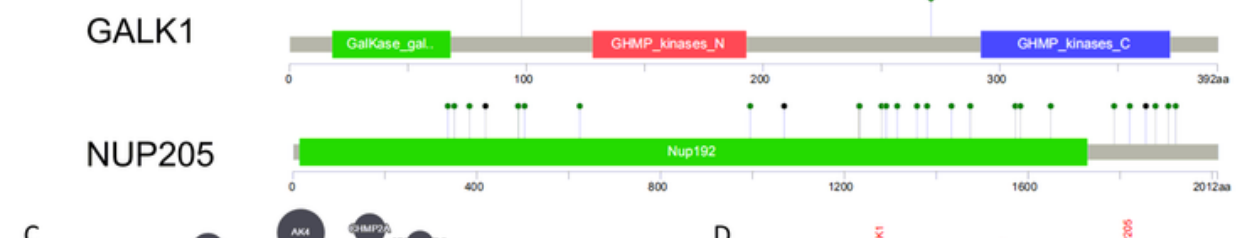

C

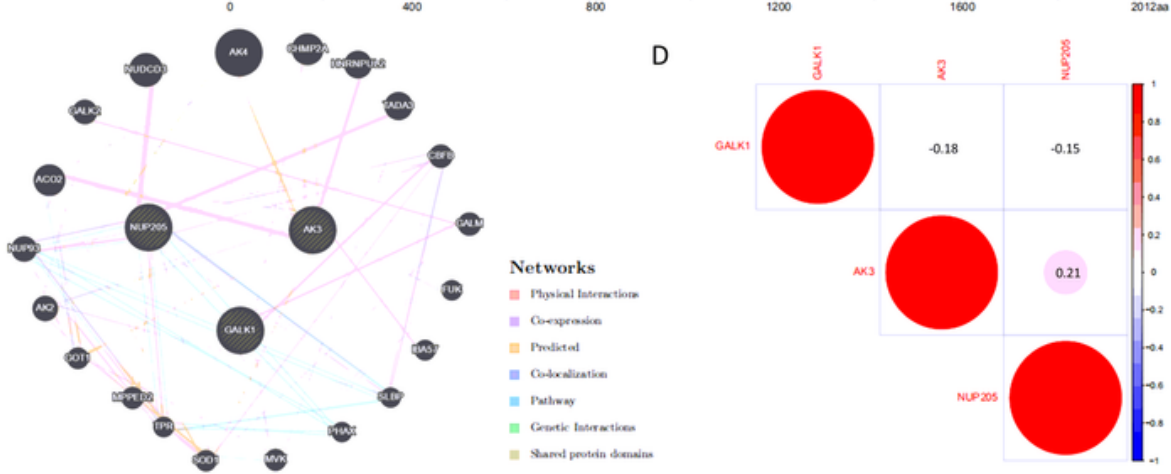

E

F
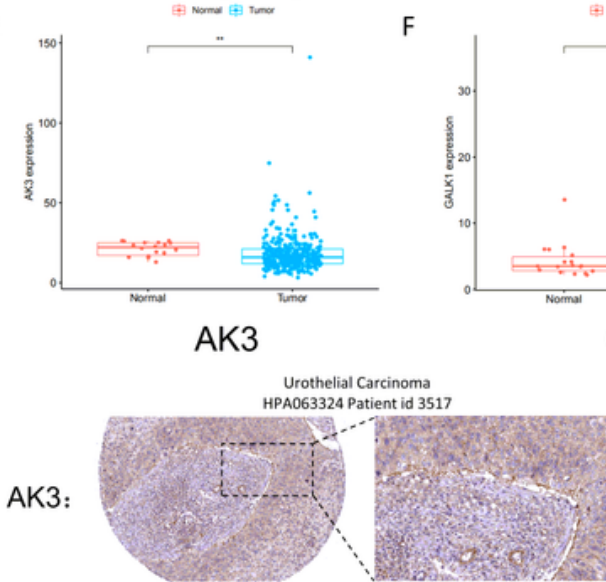

由 Nome is lom

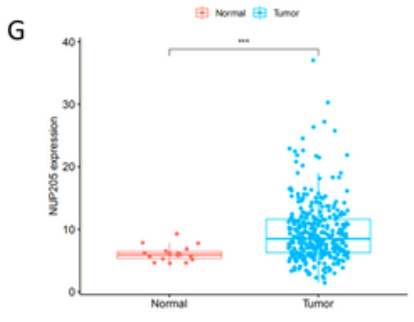

AK3

GALK1

NUP205

H

Urothelial Carcinoma
HPA063324 Patient id 351 ?

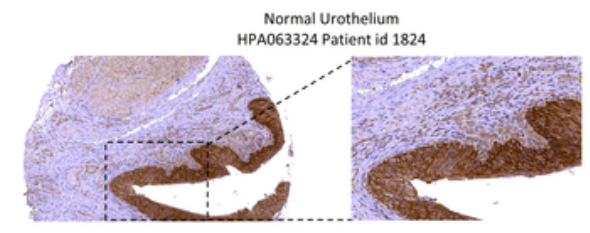

AK3:

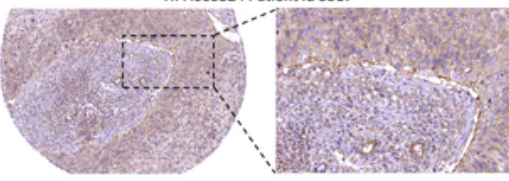

A007094 Patient id 192

Urothelial Carcinoma
HPA007094 Patient id 1723
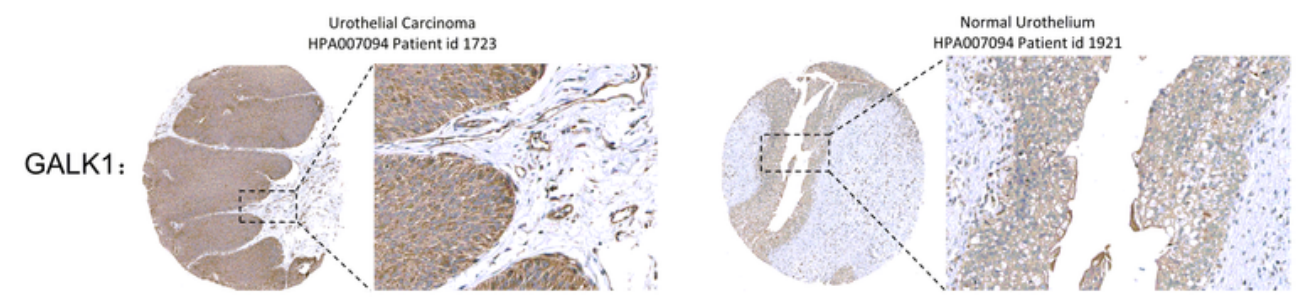

\section{Figure 5}

The detail regulatory network and gene expression about the three glycolysis related gene signature. $a, b$ ) The alteration about the three genes in proportion and the possible sites were explored with the use of the cBioPortal database. c) From the GeneMANIA we successfully built a protein-protein interaction network including these three genes. d) The interaction between the three genes in the dataset from TCGA were analyzed with the cutoff of 0.3. e-g) The expressions of these three genes from training cohort BLCA 
tissue are compared with normal tissues and $h$ ) we also downloaded IHC staining of both AK3 and GALK1 protein in normal urothelial and bladder cancer tissues from the Human Protein Atlas database.

Figure 6 :

A

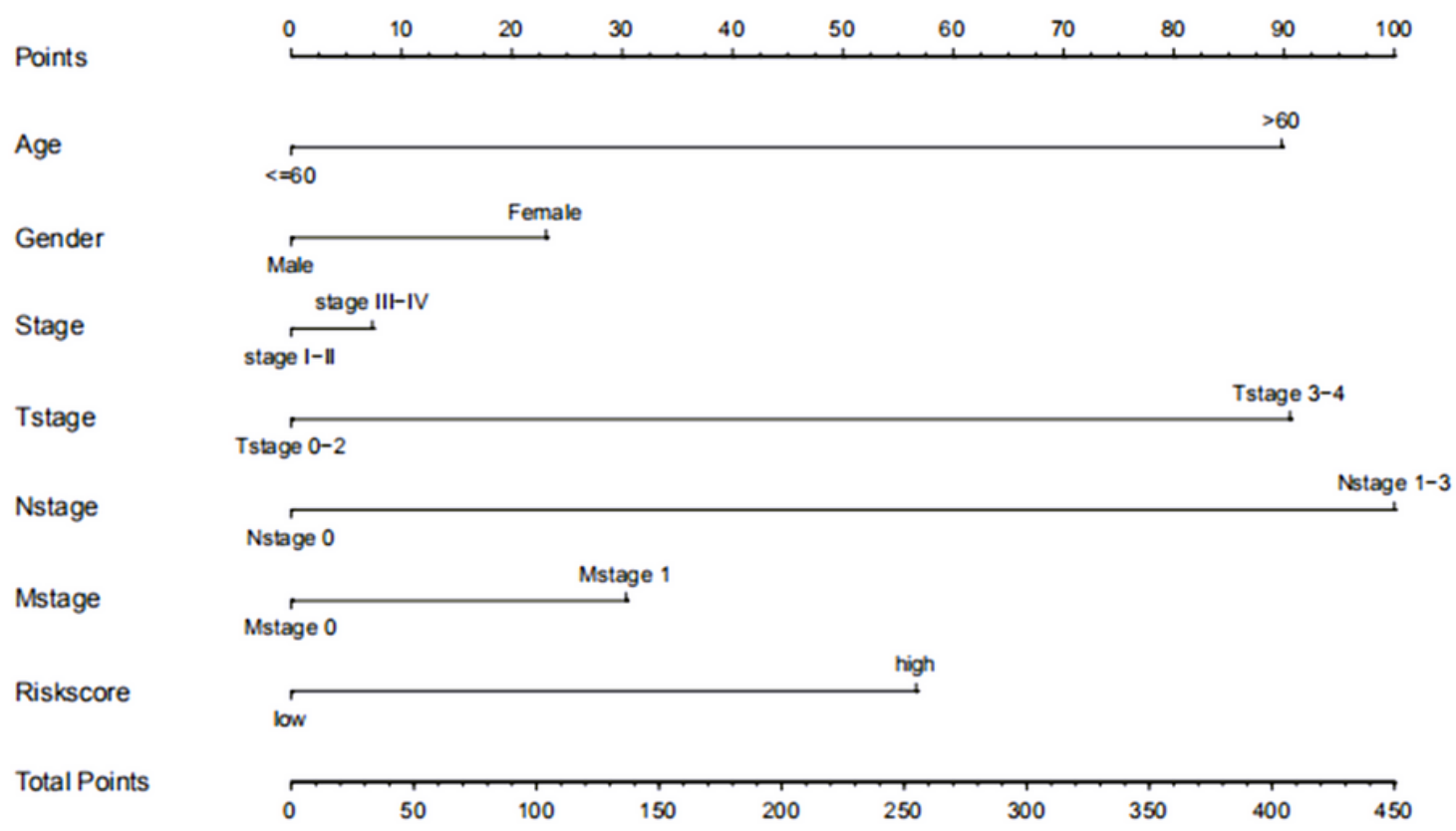

3-Year Survival

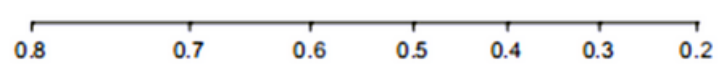

5-Year survival
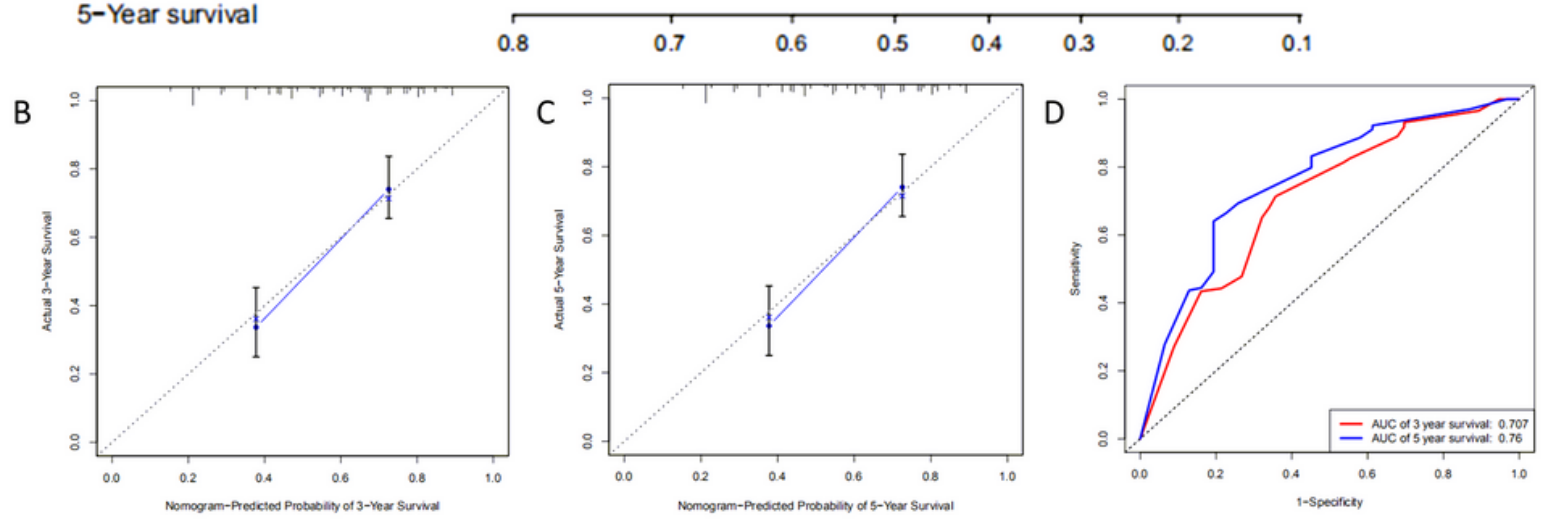

\section{Figure 6}

a) Nomogram containing clinical factors and risk scores incorporated with the 3-mRNA signature for predicting 3-and 5-year OS based on TCGA. b,c) The calibration plots for predicting patient OS at 3-and 5 -years. Probability of survival predicted by nomogram is plotted on the X-axis while actual survival is plotted on the Y-axis. d) Time-dependent ROC curves rely on the nomogram from training cohort for OS at 3- and 5-years.

\section{Supplementary Files}


This is a list of supplementary files associated with this preprint. Click to download.

- Sfigure.tif 